\title{
Síndrome compartimental agudo como complicación de un loxoscelismo cutáneo edematoso
}

\author{
Acute compartment syndrome as a complication of cutaneous loxoscelism mainly \\ edematous
}

Rocío Jara ${ }^{1}$, Celso Castillo ${ }^{1}$, María Valdés ${ }^{1}$, Claudia Albornoz ${ }^{1}$, Patricio Andrades ${ }^{1}$, Stefan Danilla ${ }^{1}$, Sergio Sepúlveda ${ }^{1}$ y Cristian Erazo ${ }^{1}$

`Unidad de Cirugía Plástica, Departamento de Cirugía, Hospital Clínico de la Universidad de Chile. Santiago, Chile.

Los autores declaran no tener conflicto de interés ni haber recibido apoyo financiero para el diseño y publicación de este estudio.

Recibido: 28 de mayo de 2019 / Aceptado: 30 de marzo de 2020

\section{Resumen}

El síndrome compartimental agudo (SCA) es el aumento de la presión en un espacio osteofascial cerrado. Esto reduce la presión capilar bajo el nivel necesario para la viabilidad de los tejidos del compartimento. El SCA de mano como complicación de un loxoscelismo cutáneo (LC) de predominio edematoso es muy poco frecuente. Presentamos el caso de una mujer de 22 años que presentó un SCA de la mano secundario a un LC y que requirió tratamiento quirúrgico de urgencia mediante una fasciotomía dorsal y palmar.

Palabras clave: Loxoscelismo cutáneo; síndrome compartimental; Loxosceles spp.; araña del rincón; mordedura araña.

\section{Introducción}

$\mathrm{E}$ 1 síndrome compartimental agudo (SCA) de la extremidad es un conjunto de síntomas y signos clínicos secundarios al aumento de la presión intersticial en un compartimento anatómico, determinando la disminución de la perfusión de los tejidos y, en consecuencia, la muerte celular ${ }^{1}$. Es un cuadro de inicio brusco, progresivo y grave. El pilar del tratamiento es la descompresión quirúrgica del compartimento. La magnitud y duración del SCA influyen directamente en la viabilidad tisular, por lo que se recomienda realizar fasciotomías precozmente $^{1,2}$. El SCA de la mano es una entidad poco frecuente. Las etiologías asociadas han sido reportadas principalmente a través de series clínicas y casos clínicos ${ }^{3,4}$. Entre éstas, la mordedura de araña es una causa extremadamente infrecuente ${ }^{5}$.

\begin{abstract}
Acute compartment syndrome (ACS) is the increase of pressure in a closed osteofascial space. This reduces capillary perfusion below the level necessary for tissue viability. Injury could be irreversible if proper treatment is not performed. Hand ACS secondary to cutaneous loxoscelism with edematous predominance is extremely infrequent. We present a clinical case of a 22 -year-old patient who started a hand compartment syndrome secondary to cutaneous loxoscelism (CL), requiring emergency surgical treatment with dorsal and palmar fasciotomy.

Keywords: cutaneous loxoscelism; compartment syndrome; Loxosceles spp; brown recluse spider; spider bite.
\end{abstract}

El loxoscelismo es la manifestación clínica en relación al veneno por la mordedura de araña del género Loxosceles, cuya especie endémica más frecuente en Chile y Sudamérica es Loxosceles laeta, conocida también como 'araña de rincón' ${ }^{6}$. Su mordedura se produce con mayor frecuencia en períodos cálidos de primavera y verano, y en ambientes oscuros tanto domésticos como al aire libre 7 . En nuestro país, el loxoscelismo es un problema de salud pública, debido a la prevalencia y gravedad de los casos identificados ${ }^{8}$. Existen dos formas clínicas del loxoscelismo", el loxoscelismo cutáneo (LC) que corresponde a $85 \%$ de los casos y el loxoscelismo cutáneo visceral (LCV) que corresponde a $15 \%$ de los $\operatorname{casos}^{10}$. El LC se manifiesta, a nivel local o regional, con dolor y eritema, evolucionando frecuentemente hacia la formación de una úlcera necrótica (placa livedoide) en el sitio de la mordedura ${ }^{9,10}$. 


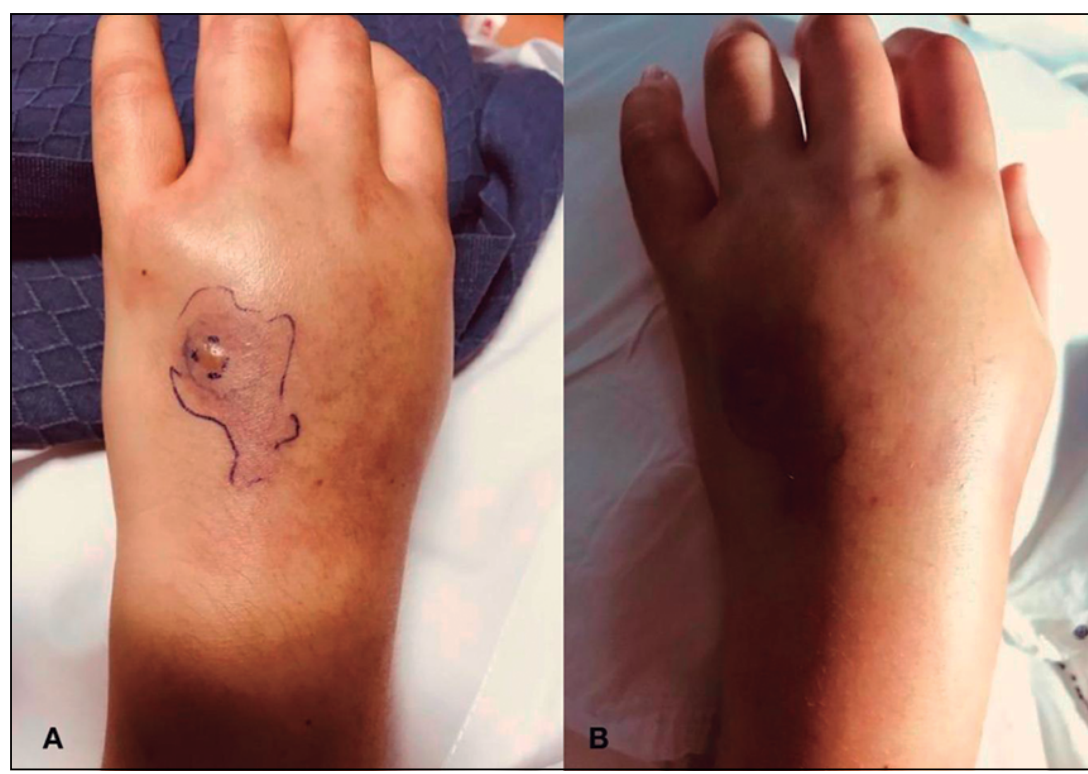

Figura 1. A. Fotografía $72 \mathrm{~h}$ de evolución de mordedura de araña del género Loxosceles spp. Se identifican compromiso edematoso de falanges, mano y muñeca izquierda. En región dorsal de mano se identifica placa livedoide inicial con flictena central en sitio de mordedura. B. Imagen preoperatoria 72-96 h posterior a la mordedura de araña. Se observa marcado edema de falanges y mano izquierda. Placa livedoide sin cambios.

El loxoscelismo cutáneo (LC) edematoso es una variedad poco frecuente, con cerca del $4 \%$ de los casos. En esta forma de presentación el factor cardinal es el edema, que puede o no estar acompañado de la lesión necrótica ${ }^{10-13}$.

El SCA es una complicación muy infrecuente del LC edematoso. Se comunica el caso de una paciente que evolucionó con un SCA de la mano secundario a un LC edematoso.

\section{Caso clínico}

Mujer de 22 años, sin antecedentes mórbidos, que consultó en el servicio de urgencias por dolor en el dorso de la mano izquierda secundario a una mordedura de araña, mientras se encontraba realizando labores de aseo. Al momento del ingreso, la paciente no portaba el arácnido para su revisión, pero lo describió como una araña alargada, de color café oscuro y de aspecto piloso, compatible con L. laeta. Al examen físico destacaba en el dorso de la mano una lesión eritematosa de $1 \mathrm{~cm}$ de diámetro aproximadamente, solevantada, delimitada por un borde hiperémico sin necrosis, de seis horas de evolución. Por las características de la lesión y la descripción de la araña, se diagnosticó un LC sin compromiso visceral, y fue dada de alta con indicación de frío local, prednisona $1 \mathrm{mg} / \mathrm{kg} /$ día y antihistamínicos orales. Consultó nuevamente a las $48 \mathrm{~h}$ por un aumento del dolor en la mano y el antebrazo. $\mathrm{Al}$ examen físico destacaba una progresión del eritema y edema hasta la fosa antecubital y una leve extensión de la placa livedoide en comparación a la primera consulta (Figura 1A). Se decidió su internación para el manejo del dolor. Se realizó una ecografía de tejidos blandos, identificándose un extenso edema del tejido celular subcutáneo, sin colecciones. Se optimizó el esquema analgésico, se mantuvieron los corticoesteroides y antihistamínicos y se inició terapia antibacteriana. A las $12 \mathrm{~h}$ de su ingreso hospitalario evolucionó con una progresión del dolor de predominio distal (EVA 10) y parestesias en las falanges. Requirió infusión continua intravenosa de antiinflamatorios no esteroidales y opiáceos. Evolucionó con edema a tensión de la mano, dolor pasivo a la movilización de las falanges, extensión de las articulaciones metacarpofalángicas y leve flexión de las articulaciones interfalángicas, con persistencia del llene capilar y pulso radial sin alteraciones (Figura 1B). La paciente fue evaluada por un equipo multidisciplinario compuesto por cirujanos plásticos, vasculares, traumatólogos y especialistas en control del dolor, diagnosticándose un SCA de la mano. Desde su ingreso hospitalario se había indicado elevación de la extremidad y frío local, sin respuesta clínica. Frente al diagnóstico de una SCA de mano se decidió un tratamiento quirúrgico precoz. Se realizaron fasciotomías descompresivas dorsales del compartimento interóseo y tenar (Figura 2. A1) y una liberación del túnel carpiano mediante incisión volar (Figura 2. A2). Se ubicaron suturas irreabsorbibles en la dermis para realizar afrontamiento progresivo en el postoperatorio (Figura 2. B1-B2). En el intraoperatorio se identificaron cambios isquémicos a nivel del compartimento muscular, los que se recuperaron prontamente luego de las fasciotomías. Posterior a la cirugía se constató una disminución del dolor y edema de la extremidad. Se inició la rehabilitación de la extremidad en el segundo día postoperatorio, logrando la movilidad con buena tolerancia al dolor. A las 72 h se inició el afrontamiento progresivo, logrando el cierre completo de las incisiones al quinto día postoperatorio (Figura 2. C1-C2). La paciente fue dada de alta el séptimo día postoperatorio con una adecuada movilidad de la mano. La herida fue manejada con curaciones avanzadas y no requirió cobertura quirúrgica (Figura 2. D1-D2).

\section{Discusión}

El SCA de mano es una urgencia quirúrgica poco frecuente y puede ser desencadenado por múltiples causas. Entre ellas se encuentran: fractura, trauma, quemaduras, infiltración intravenosa, compresión externa, infección, hemorragia, envenenamiento por serpientes y picaduras de insectos ${ }^{3,4}$.

En las extremidades, lo más frecuente es el antecedente de fractura; sin embargo, hasta $30 \%$ de los pacientes no 
tienen esta condición ${ }^{14}$. La ausencia de fractura lleva a que el diagnóstico se realice en forma tardía y, al momento de la fasciotomía, los pacientes tengan significativamente mayor necrosis muscular, en comparación a aquellos en los que se identifica el antecedente de fractura $(20 \%$ versus $8 \%$; respectivamente), agravando el pronóstico de $\operatorname{los} \operatorname{casos}^{14}$. Sin embargo, estos hallazgos pueden no ser extrapolables al SCA de la mano debido a la baja incidencia y heterogeneidad de esta patología.

McKnight $^{5}$ reportó el caso de un paciente de 18 meses con un SCA de mano secundario a una picadura de un mosquito, requiriendo fasciotomías de la mano y liberación profiláctica del túnel carpiano. La extensa reacción inflamatoria a nivel del compartimento muscular fue considerada el factor desencadenante en la evolución del paciente.

El SCA asociado a LC ha sido reportado en relación mordeduras de araña en la región periorbitaria. En una serie de 29 pacientes con mordeduras de araña del género Loxosceles spp., Hubbard y cols. ${ }^{15}$ reportaron dos casos de SCA orbitario. Jarvis y cols. ${ }^{12}$, comunicaron el caso de una paciente de 8 años con LC edematoso que evolucionó con un SCA orbitario y que requirió descompresión quirúrgica. La extensa progresión edematosa del LC pudo ser una de las causas desencadenantes del SCA de mano en nuestra paciente.

En nuestro país se reportan cerca de 250 casos por año de LC, de estos el LC edematoso es de presentación muy poco frecuente. Aún más excepcional, es su complicación con SCA, por esto consideramos relevante presentar este caso, pese a que no se obtuvo el espécimen, lo que solo se logra en $60 \%$ de los casos. La descripción realizada en nuestra paciente resultó concordante con una araña del rincón como posible agente causante de la lesión ${ }^{16}$.

La paciente presentó dolor, eritema y edema en la mano, lo que se encuentra dentro de las ubicaciones más frecuentes de mordedura. Según lo descrito por Schenone, en los casos de LC de predominio edematoso las mordeduras serían más frecuentes en cara, cuello y extremidades proximales; opuesto a esto, el caso que presentamos comprometió la extremidad hacia distal lo que es otro punto relevante en este caso como presentación atípica ${ }^{12}$. A las pocas horas de evolución se observó un halo violáceo en relación al sitio de mordedura y posteriormente, a las 48 h de evolución, se inició la conformación de la placa livedoide. Destacó en su evolución clínica un dolor progresivo en intensidad, desproporcionado y refractario a analgésicos de alta potencia, asociado a la presencia de parestesias distales de la extremidad. Lo anterior, sumado a los hallazgos en el examen físico: edema tenso, dolor a la movilización pasiva e impotencia funcional, hicieron sospechar un SCA de la mano, lo que por su ubicación plantea un desafío en el manejo.

Clásicamente los signos clínicos han sido utili-

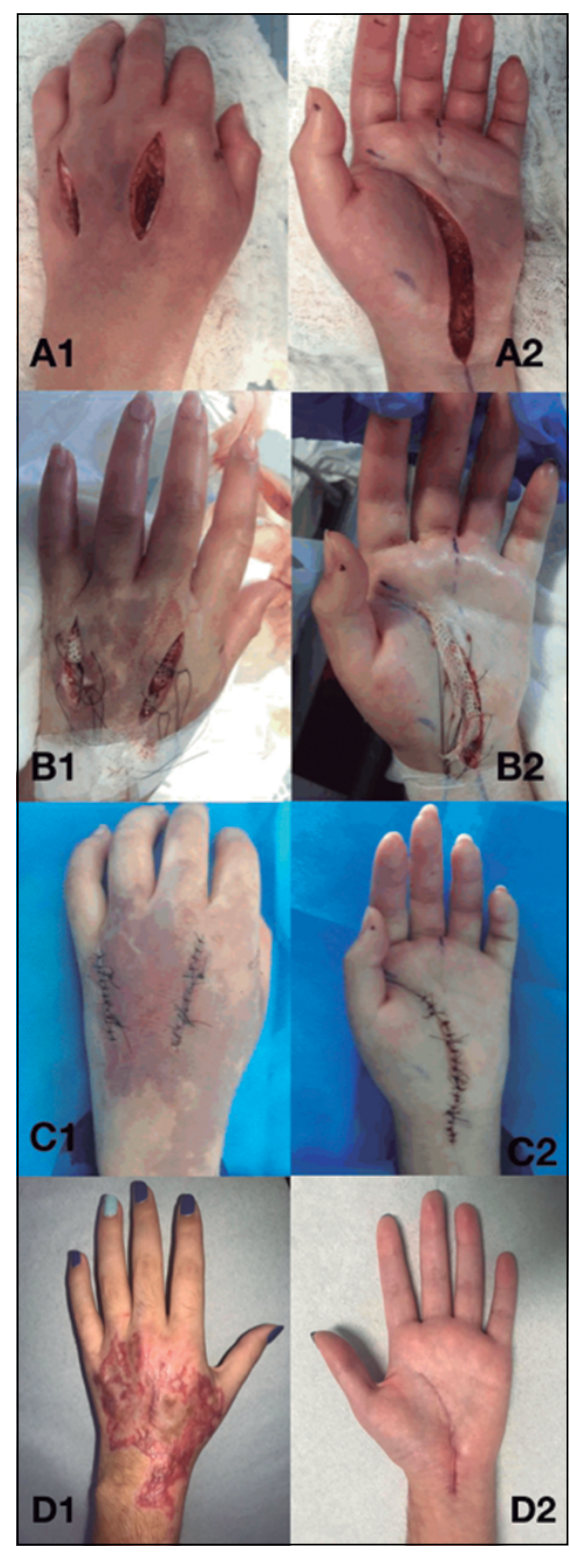

Figura 2. A1-2. Intraoperatorio. Fasciotomías dorsales y ventrales, respectivamente. B1-2. Primer día postoperatorio. Disminución de edema mano izquierda y evolución de placa livedoide. Heridas quirúrgicas con suturas previo a afrontamiento progresivo. C1-2. Quinto día postoperatorio. Heridas quirúrgicas suturadas. Mayor extensión de placa livedoide. D1-2. Quinto mes postoperatorio. En región de placa livedoide se observa cicatrización completa y activa de herida. zados para describir los hallazgos característicos en un $\mathrm{SCA}^{17,18}$; sin embargo, estos son signos tardíos y consistentes con un daño irreversible, por lo que existe acuerdo en reconocer la desproporción y progresión del dolor como los hallazgos precoces más frecuentes en $\mathrm{SCA}^{2,3,19}$. Ouellette ${ }^{4}$, sugiere que los signos más consistentes con SCA son: mano tensa y edematosa, postura de extensión de las articulaciones metacarpofalángicas y flexión de las articulaciones interfalángicas proximales y distales; y dolor a la extensión pasiva de los músculos del compartimento implicado.

Con el fin de objetivar la presión intracompartimental se ha utilizado la manometría arterial y dispositivos 


\section{- Grei canc clinio}

comerciales. No obstante, estos métodos han sido validados como complementos al diagnóstico en el entorno hospitalario frente a la duda diagnóstica ${ }^{17,20}$.

En nuestra comunicación, debido a la elevada sospecha clínica de un SCA se optó por realizar un tratamiento quirúrgico inmediato.

La lesión neurovascular es una de las principales complicaciones asociadas a la descompresión quirúrgica, por lo que debe ser realizada con precaución ${ }^{4,21}$. En este reporte, la fasciotomía se realizó identificando los planos anatómicos para evitar la lesión del nervio mediano durante la liberación del túnel carpiano y con liberación digital durante las fasciotomías volares.

El manejo no quirúrgico inicial ha sido planteado como una alternativa conservadora con el fin de disminuir la morbilidad asociada a la fasciotomía ${ }^{3}$. Dado que es ampliamente reconocido que un retraso en el diagnóstico y en la descompresión quirúrgica comprometen el pronóstico de estos pacientes, el rol del manejo no quirúrgico es limitado a casos de irrecuperabilidad de la extremidad, ya sea por error médico en el diagnóstico, o bien por condiciones que limiten el tratamiento quirúrgico del paciente. El error médico en el SCA puede traer consecuencias médico-legales, lo que ha sido previamente reportado. Pese a que las causas legales relacionadas al SCA son poco frecuentes, pueden cursar con un alto costo de indemnización. Una pobre comunicación médicopaciente y el retraso en la realización de fasciotomías son las principales variables involucradas en el aumento de los costos de indemnización ${ }^{22}$. Debido a esto, sugerimos que frente a la sospecha de un SCA se debe realizar una evaluación multidisciplinaria para aproximar el diagnóstico, y una vez realizado, debe involucrar una conducta quirúrgica oportuna ${ }^{23}$.

La principal morbilidad funcional asociada al SCA de mano es la contractura muscular intrínseca generada por los cambios fibróticos derivados de la isquemia muscular persistente. La probabilidad de presentar esta secuela disminuye con el diagnóstico de un SCA en las etapas iniciales ${ }^{3,4,21}$. En nuestro caso, el diagnóstico precoz e inicio temprano de la rehabilitación funcional permitieron una adecuada recuperación de la extremidad.

El LC de predominio edematoso como causa de un SCA de mano es infrecuente, pero debe ser considerado en una zona endémica de Loxosceles spp. El pilar del manejo es la descompresión quirúrgica de los compartimentos comprometidos con el fin de restaurar la perfusión de la extremidad y evitar secuelas irreversibles.

\section{Referencias bibliográficas}

1.- Finkelstein J A, Hunter G A, Hu R W. Lower limb compartment syndrome: course after delayed fasciotomy. J Trauma 1996; 40: 342-4.

2.- Vaillancourt C, Shrier I, Falk M, Rossignol M, Vernec A, Somogyi D. Quantifying delays in the recognition and management of acute compartment syndrome. Can J Emerg Med 2001; 3: 26-30.

3.- Rubinstein A, Ahmed I, Vosbikian M. Hand Compartment Syndrome. Hand Clin 2018; 34: 41-52. doi: 10.1016/j.hcl.2017.09.005.

4.- Ouellette E A, Kelly R. Compartment syndromes of the hand. J Bone Joint Surg Am 1996; 78: 1515-22.

5.- McKnight A J, Koshy J C, Xue A S, Shetty M, Bullocks J M. Pediatric compartment syndrome following an insect bite: a case report. Hand $(\mathrm{N}$ Y) 2011; 6: 337-9. doi: 10.1007/s11552-0119338-4.

6.- Schenone H, Saavedra T, Rojas A, Villarroel F. Loxoscelismo en Chile. Estudios epidemiológicos, clínicos y experimentales. Rev Inst Med Trop. Sao Paulo 1989; 31: 40315.

7.- Gertsch W, Ennik F. The spider genus Loxosceles in North America, Central America, and the West Indies (Araneae, Loxoscelidae). Bull American Mus Nat Hist 1983; 175: 264360 .

8.- Guía para el Manejo de Mordedura de Araña de los Rincones - Loxosceles laeta Chile, 2016. Disponible en: http://cituc.uc.cl/ images/PROTOCOLO-LOXOSCELESMINSAL-2016.pdf

9.- Swanson D L, Vetter R S. Bites of brown recluse spiders and suspected necrotic arachnidism. N Engl J Med 2005; 352: 700-7.

10.- Manríquez J J, Silva S. Loxoscelismo cutáneo y cutáneo-visceral: Revisión sistemática. Rev Chilena Infectol 2009; 26: 420-32. doi: /S071610182009000600004.

11.- De la Barra P, Vial V, Labraña Y, Álvarez A, Seguel H. Cutaneous loxoscelism mainly edematous: a case report. Rev Chilena Infectol 2015; 32: 467-71. doi: 10.4067/S071610182015000500017.

12.- Schenone H. Cutaneous loxoscelism with edematous predominance. Bol Chil Parasitol 1998; 53 (3-4): 78-83.

13.- Schenone H. Cuadros tóxicos producidos por mordeduras de araña en Chile: latrodectismo y loxoscelismo. Rev Med Chile 2003; 131: 437 44.

14.- Hope M J, McQueen M M. Acute compartment syndrome in the absence of fracture. J Orthop Trauma 2004; 18: 220-4.

15.- Hubbard J J, James L P. Complications and outcomes of brown recluse spider bites in children. Clin Pediatr (Phila) 2011; 50: 252-8. doi: 10.1177/0009922810388510.

16.- CITUC. Araña de rincón. Disponible en: http:// cituc. saluduc.cl/link.cgi/ABC/A/arana_de_ rincon.act. Fecha de acceso: 27 de julio de 2016.

17.- Boody A R, Wongworawat M D. Accuracy in the measurement of compartment pressures: a comparison of three commonly used devices. J Bone Joint Surg Am 2005; 87: 2415-22.

18.- Leversedge F J, Moore T J, Peterson B C, Seiler J D $3^{\text {rd }}$. Compartment syndrome of the upper extremity. J Hand Surg Am 2011; 36 : 544-59. doi: 10.1016/j.jhsa.2010.12.008.

19.- Uliasz A, Ishida J T, Fleming J K. Comparing the methods of measuring compartment pressures in acute compartment syndrome. Am J Emerg Med 2003; 21: 143-5.

20.- Hammerberg E M, Whitesides T E Jr, Seiler J $\mathrm{G} 3^{\text {rd }}$. The reliability of measurement of tissue pressure in compartment syndrome. J Orthop Trauma 2012; 26: 24-31. doi: 10.1097/ BOT.0b013e31822908cf.

21.- Griffiths D. Volkmann's ischaemic contracture. Br J Surg 1940; 2 (4149): 66-7. https://www.ncbi.nlm.nih.gov/pmc/ articles/PMC2178833/pdf/brmedj04101-0030c. pdf

22.- Bhattacharyya T, Vrahas M. The medical-legal aspects of compartment syndrome. J Bone Joint Surg Am 2004; 86: 864-8.

23.- Von Keudell A G, Weaver M J, Appleton P T, Bae D S, Dyer G S M, Heng M, et al. Diagnosis and treatment of acute extremity compartment syndrome. Lancet 2015; 386: 1299-310. doi: $10.1016 / \mathrm{S} 0140-6736(15) 00277-9$. 